\title{
Techniques for Transmission EBSD Mapping of Atom Probe Specimens
}

\author{
Katherine P. Rice ${ }^{1}$, Yimeng Chen ${ }^{1}$, Ty J. Prosa ${ }^{1}$, David J. Larson ${ }^{1}$, Matt Nowell ${ }^{2}$ and Mark P. \\ Stoykovich ${ }^{3}$ \\ 1. CAMECA Instruments Inc., Madison, WI 53711 USA \\ 2. EDAX, Draper, UT 84020 USA \\ ${ }^{3 .}$ Department of Chemical and Biological Engineering, University of Colorado, Boulder, CO 80309 \\ USA
}

Transmission Electron Back Scatter Diffraction (t-EBSD)/Transmission Kikuchi Diffraction (TKD) enables crystallographic identification in samples of much smaller size, and with improved spatial resolution, as compared to conventional reflection EBSD. In t-EBSD, the elimination of a severe tilt angle and the collection of primarily low-loss electrons keeps the probe small enough for mapping resolution down to approximately $2 \mathrm{~nm}$ [1]. Atom probe tomography (APT) relies on needle shaped specimens with an apex radius of $\sim 50 \mathrm{~nm}$ in order to produce the critical electric field for evaporating ions. Such needle shapes are then ideally sized for characterization with t-EBSD. Atom probe data can provide information about segregation at grain boundaries. Combination of this chemical information with crystallographic information from t-EBSD provides a more complete picture of the chemical composition and the microstructure of the sample [2].

Here we present strategies to optimize t-EBSD for atom probe applications, including sample holding fixtures and microscope parameters that enable the mapping of multiple specimens without the need to break vacuum. Strategies to avoid contamination of the sample during the mapping process will be presented and optimal map conditions for atom probe specimens will be discussed. Moreover, we will discuss challenges with the application of t-EBSD to the characterization of the inherently threedimensional specimens of interest for APT. Unlike conventional reflection EBSD, the mapped surface in transmission is the bottom surface [3], which can present unique difficulties in a specimen with multiple or overlapping grains that can cause overlapping or blurring of the Kikuchi patterns. We demonstrate this particular phenomenon in an APT specimen by mapping a sample with Atom Probe Assist $^{\mathrm{TM}}$ mode with side-by-side grains and introducing a 90 degree rotation, such that only one pattern is visible. One critical element in successfully mapping an APT specimen is overcoming the FIBinduced $\mathrm{Ga}$ ion implantation which can cause loss of crystallinity in the region of interest [4]. Cleaning the surface with a low-energy ion beam [5] is demonstrated to significantly improve the image quality of the Kikuchi patterns. Figure 1 shows a typical t-EBSD map obtained from a nickel APT specimen. The inset demonstrates a typical Kikuchi pattern obtained from the specimen.

In t-EBSD, like all transmission imaging and diffraction techniques, the sample affects the achievable spatial resolution because the beam broadens and loses energy as the electrons undergo multiple scattering events to reach the exit surface. Beam broadening is particularly important when mapping atom probe specimens, where sample thicknesses may range from tens to a few hundred nanometers. Beam size changes across the cone-shaped APT specimen can be modeled to a first approximation with Monte Carlo simulations of electron trajectories. Our models [3] provide estimates of beam size and effective resolution at each point. Figure 2 shows the electron distribution at the exit surface for two different cases: (a) the beam impinging at the center of the tip $100 \mathrm{~nm}$ down the shank from the apex; and (c) the beam impinging at the center of the tip $500 \mathrm{~nm}$ down the shank from the apex. The models 
show that at a distance of $500 \mathrm{~nm}$ away from the apex, the distribution of electrons covers a considerable portion of the tip surface. This suggests that there are enough electrons to create a diffraction pattern from the entire bottom surface of the specimen at this thickness, and it may be difficult to isolate single grains during the mapping process. This conclusion is supported by the data in Figure 1, where there are non-indexed points (black regions) in the center of the specimen at the thicker portions of the tip.

\section{References}

[1] P. W. Trimby et al., Ultramicroscopy 120 (2012), p. 16.

[2] K. Babinsky et al., Ultramicroscopy 144 (2014), p. 9.

[3] K. Rice et al., J. Microscopy 254 (2014), p. 129.

[4] J. Mayer et al., MRS Bulletin 32 (2007), p. 400.

[5] K. Thompson et al., Microscopy and Microanalysis 12(S2) (2006), p. 1736.

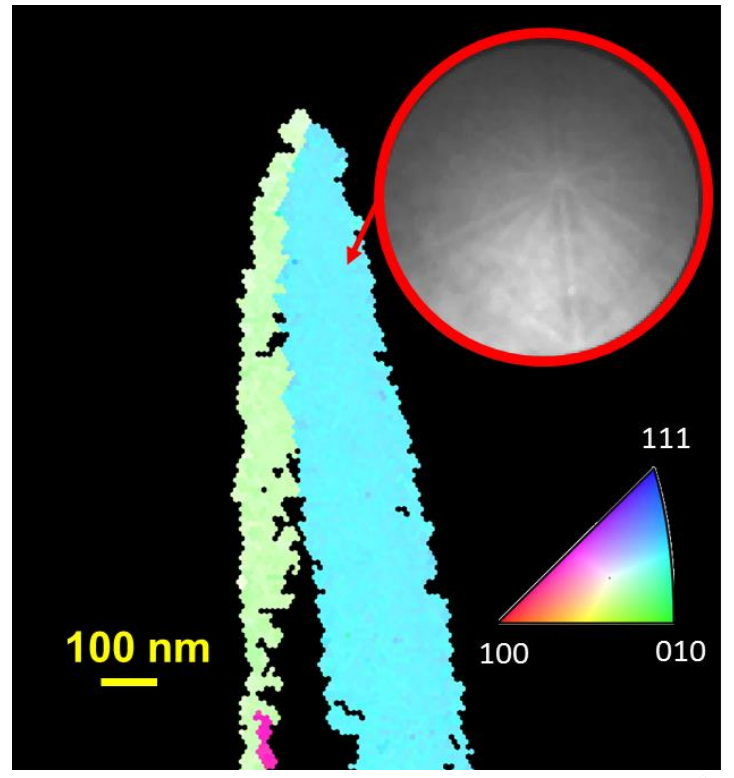

(a)

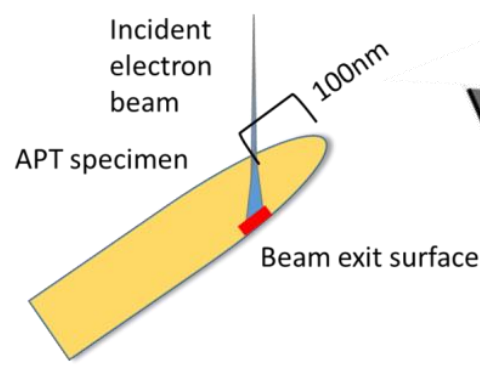

(c)

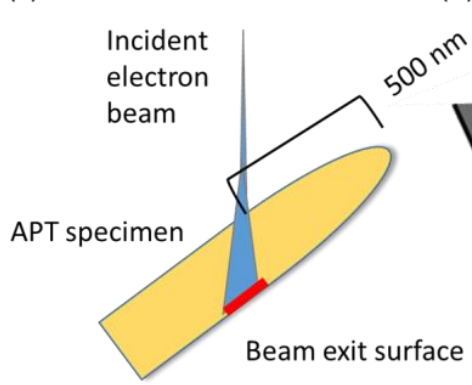

(d)
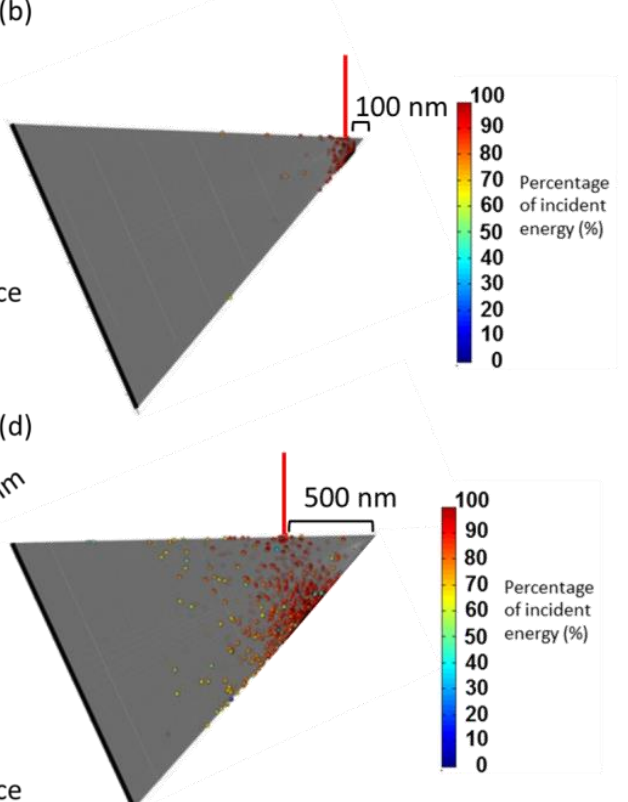

Figure 1: Orientation map with minimal cleanup of a nickel APT specimen showing a twin grain boundary. Inset shows an example Kikuchi pattern obtained from the specimen.

Figure 2 (a) and (c): Schematics of incident electron beam impinging on two locations along an APT specimen (a) $100 \mathrm{~nm}$ from the tip apex, and (c) $500 \mathrm{~nm}$ from the apex.

Figure 2 (b) and (d): Electron scattering simulations showing the location and energy of electrons exiting the bottom surface of the cone-shaped APT specimen. The electrons are colored by the percentage of incident energy retained at the exit surface. One thousand electrons were used in each simulation. 